\title{
Monitoring Salivary Immunoglobulin A Responses to Official and Simulated Matches In Elite Young Soccer Players
}

\author{
by \\ Camila G. Freitas ${ }^{1}$, Marcelo S. Aoki ${ }^{2}$, Ademir F. S. Arruda ${ }^{1}$, \\ Clóvis Franciscon ${ }^{3}$,Alexandre Moreira ${ }^{1}$
}

\begin{abstract}
The purpose of the present study was to examine SIgA responses (concentration [SIgAabs] and a secretion rate [SIgArate]) to official and simulated competitive matches in young soccer players. The sample was composed of 26 male soccer players (age $15.6 \pm 1.1 \mathrm{yrs}$, stature $177.0 \pm 6.1 \mathrm{~cm}$, body mass $70.5 \pm 5.7 \mathrm{~kg}$ ). Four soccer matches (two simulated matches [SM] and two official matches [OM]) were conducted. The matches consisted of two halves of 35 min with a 10 min rest interval. Each assessed player participated in only one SM and one OM. All matches were performed in the same week, during the competitive season, and at the same time of the day (9:00 am), separated by $48 \mathrm{~h}$. Saliva samples were collected before and after every match. The session rating of perceived exertion was reported 30 min after each match in order to determine the internal training load (ITL). A significant decrease in SIgAabs and SIgArate after OM was observed when compared to the pre-match value. In addition, the SIgArate was higher at pre-OM when compared to pre-SM. A higher ITL for OM was observed compared to SM. The current findings indicate that OM may lead to a decrease in the main mucosal immunity function parameter of young soccer players that could increase the risk of URTI. Coaches should be aware of it in order to plan appropriate training loads and recovery procedures to avoid or minimize the likelihood of upper respiratory tract infection occurrences.
\end{abstract}

Key words: SIgA, mucosal immunity, athletic performance, sports, adolescents.

\section{Introduction}

The mucosal surfaces are normally protected by a network of organized structures located in the gut, urogenital tract, oral cavity and respiratory system, collectively known as the mucosal immune system (MIS) (Gleeson and Pyne, 2000). A major component of the MIS is salivary immunoglobulin A (SIgA). In fact, a low level of SIgA has been associated with an increased risk of upper respiratory tract infection (URTI) in athletes (Bishop and Gleeson, 2009; Fahlman and Engels, 2005; Gleeson et al., 2012). Moreover, a transient fall in SIgA has been shown to be a good predictor for increased risk of URTI
(Neville et al., 2008). The role of SIgA is to prevent viral replication and to inhibit the attachment of bacteria and viruses at mucosal epithelium in the mouth, throat and upper respiratory tract (Mackinnon, 1996). Therefore, a decrease in the production and/or concentration of this antibody would represent a risk factor for subsequent URTI episodes in athletes (Fahlman and Engels, 2005).

With regard to studies on endurance athletes, the SIgA acute responses in team sport athletes have been less investigated ( $\mathrm{He}$ et al., 2010; Koch et al., 2007; Moreira et al., 2011a; Moreira et al., 2013a). Results from these

1 - School of Physical Education and Sport, Department of Sport, University of São Paulo, São Paulo, Brazil.

2- School of Arts, Sciences and Humanities, University of São Paulo, São Paulo, Brazil.

3. Desportivo Brasil, Porto Feliz, São Paulo, Brazil. 
investigations may be considered somewhat inconclusive as some showed an increment in SIgA concentration after the assessed matches while others demonstrated neither change nor decrease in SIgA concentration. However, recently, Owen et al. (2014) demonstrated a significant reduction in SIgA after completion of a high intensity training session when compared to a low intensity unit in a sample of 10 elite male professional soccer players. This result suggests that training intensity plays a key role in SIgA changes. In addition, some studies have demonstrated that the psychological stress can increase SIgA concentration during tasks that do not involve physical effort (Benham et al., 2009; Bosch et al., 2001; Zeier et al., 1996).

However, most of the aforementioned studies were conducted on young adults or professional players. Only a few studies that have examined the SIgA responses to training or competition in young athletes (Filaire et al., 2004; Li et al., 2012; Mortatti et al., 2012; Nieman et al., 2000). Nowadays, like adult athletes, young team sport athletes are often submitted to intense training loads and high competition demands (Hartwig et al., 2009). This population is also under continued pressure to obtain good results in their age category competitions and has to cope with the uncertainty of achieving the high-level professional setting. This scenario, therefore, imposes a great level of psychological and physiological stress to young soccer players which might not be prepared to deal appropriately with all these demands.

Among the few studies conducted with young team players, focused on SIgA response and its association with stress (Filaire et al., 2004; Li et al., 2012; Nieman et al., 2000), Mortatti et al. (2012) monitored young soccer players through an official competition which included 7 matches played over 20 days, and reported a significant relationship between the SIgA decrease and the incidence of URTI symptoms, as the team advanced towards the final stages of the competition. Indeed, Moreira et al. (2014) examined the effect of a 21 week competitive season divided into a pre-season (12 weeks), a competitive season (7 weeks) and detraining (2 weeks) on salivary cortisol, SIgA and upper respiratory tract infection (URTI) symptoms in 34 pre-adolescent male soccer players. The results of the study showed a significant increase in a SIgA secretion rate and a decrease in URTI symptoms after the 2 week detraining period, suggesting that the accumulated training loads and competitive demands negatively affected the mucosal immunity of youth players and that a short-prophylactic period may attenuate mucosal immunosuppression related to URTI. Additionally, Nazem et al. (2011) showed a significant decrement in SIgA concentration after the most difficult matches in young female handball athletes assessed over successive matches.

Collectively, these findings suggest that intense training loads and high competition demands, as well the importance and difficulty of the match, may affect the responses of mucosal immunity. However, up to now, no previous investigations have compared the SIgA responses to simulated matches (SM) and official matches $(\mathrm{OM})$ in elite young soccer players. The advance of this knowledge could aid coaches and staff members to prescribe appropriate training loads and recovery periods in order to minimize the risk of the reduced mucosal immune function. These data would also contribute to a deeper understanding of players' preparedness for training, competition and performance. Therefore, the purpose of the present investigation was to examine SIgA responses (absolute concentration and secretion rate) to OM and SM in young soccer players. It was hypothesized that OM would lead to a greater decrement in SIgA responses due to its higher psychophysiological stress.

\section{Material and Methods}

\section{Participants}

Forty-four soccer players initially participated in the study and completed two SM. However, only data from 26 players who participated in both OM and SM were retained for analysis. The sample was composed of 26 male soccer players (age $15.6 \pm 1.1$ yrs, body height $177.0 \pm 6.1 \mathrm{~cm}$, body mass $70.5 \pm 5.7 \mathrm{~kg}$ ). Usually, the assessed players were involved in a schedule training program which consisted of 10 to 12 sessions per week, with duration of 90-120 min, including strength and conditioning sessions, sprints and repeated-sprint bouts as well as intermittent running exercises, soccer-specific drills and small-sided games. 
The investigated players were living at the training facilities of the club during the period of the investigation. Therefore, they shared accommodations, had the same meals being served at the same time, and were also submitted to similar recovery times after daily training sessions. This situation provided an appropriate environment control, therefore, reducing factors which could influence the acute SIgA responses from the investigated matches, such as nutrition status, exposition to pathogens, recovery from training and $\mathrm{OM}$, and awakening time. After approval of the Research Ethics Committee of the School of Physical Education and Sport, University of São Paulo, the experimental protocols were explained in detail. Written informed consent was obtained from each participant and their respective parents or guardians.

\section{Procedures}

Four soccer matches (two SM and two $\mathrm{OM}$ ) were conducted. The matches were composed of two halves of $35 \mathrm{~min}$ with a $10 \mathrm{~min}$ rest interval. Each assessed player participated in only one SM and one OM. All matches were performed in the same week, during the competitive season and at the same time of the day $(9: 00 \mathrm{am})$, separated by $48 \mathrm{~h}$. Data from the two SM and the two OM were pooled for analysis. Saliva samples were collected before and after each match. The session rating of perceived exertion (session-RPE) was reported $30 \mathrm{~min}$ after each match in order to monitor the internal training load (ITL).

\section{Measures}

\section{Internal Training Load (ITL)}

A session-RPE score was assessed after each match, in order to quantify the ITL, as proposed by Foster (1998). Briefly, each player rated the training session using the CR-10 sliding scale, where $0=$ nothing at all, and $10=$ very, very hard (maximal). These data were collected $30 \mathrm{~min}$ after each match to ensure that the perceived effort was based on the entire game rather than the last effort intensity. In order to determine the ITL expressed in arbitrary units (AU), the product of session duration (min) and the RPE score (CR10) rated by the player was used. All assessed players were familiarized with the CR-10 scale, as it had been used routinely during their training program.
Saliva collection

Athletes provided saliva samples approximately $10 \mathrm{~min}$ before the pre-match warm-up; post-match saliva samples were collected within 10-15 $\mathrm{min}$ of the end of the SM and OM. The participants abstained from consuming food and caffeine-containing products for at least $2 \mathrm{~h}$ before the collection of saliva. After rinsing their mouth with distilled water, athletes were seated with eyes open, the head tilted slightly forward and making minimal orofacial movement. Unstimulated saliva was collected into sterile $15 \mathrm{ml}$ centrifuge tubes over a $5 \mathrm{~min}$ period and then stored at $-80^{\circ} \mathrm{C}$ until assayed for SIgA concentration. The tubes were reweighed before analysis so that saliva volume could be estimated in accordance with the procedure adopted by Moreira et al. (2013b). Saliva density was assumed to be $1.00 \mathrm{~g} \cdot \mathrm{mL}^{-1}$. The recovery interval from the last bout of exercise was $24 \mathrm{~h}$.

SIgA assays

SIgA concentration was measured in duplicate by an enzyme-linked immunosorbent assay (ELISA; s-IgA EIA kit, ALPCO Diagnostics, Salem, MA, USA) USA). Saliva samples were thawed, centrifuged at 1,630 $\mathrm{g}$ for $10 \mathrm{~min}$, and the supernatant was diluted $(1: 2,000)$ in the ELISA wash buffer. Subsequently, $100 \mathrm{~mL}$ of calibrators and diluted saliva samples were added to microtiter wells (precoated with polyclonal rabbit anti-human IgA) and incubated for $1 \mathrm{~h}$, with constant shaking, at room temperature. After incubation, the plate was aspirated and washed 5 times with $250 \mathrm{~mL}$ of ELISA wash buffer to remove all unbound substances. Then, $100 \mathrm{~mL}$ of peroxidase-labeled mouse anti-IgA conjugated was added to each well on the microtiter plate. After incubating the plate for $1 \mathrm{~h}$, with constant shaking at room temperature, the contents of the plate were decanted and washed 5 times with 250 $\mathrm{mL}$ of the ELISA wash buffer to remove all unbound substances. After washing, $100 \mathrm{~mL}$ of tetramethylbenzidine (TMB) substrate solution was added and incubated for $5 \mathrm{~min}$ at room temperature with no mixing. This enzyme acted on the substrate and caused a blue color to appear in proportion to the amount of the peroxidase present. Finally, $50 \mathrm{~mL}$ of the stop solution was added to the wells and the optical density was read on the plate reader at $450 \mathrm{~nm}$. A yellow color was formed after the stop solution was added. 
The amount of color detected was directly proportional to the amount of SIgA present. From a calibration curve (optical density vs. IgA concentration of the calibrators), the concentration of SIgA (SIgAabs - micrograms per milliliter) in each sample was interpolated. The SIgA secretion rate (SIgArate - micrograms per minute) was calculated by multiplying the absolute SIgAabs by a salivary flow rate (milliliters per minute), in accordance with the procedure adopted in previous studies (Moreira et al., 2012b, 2013b).

\section{Statistical analysis}

Data were analyzed using Statistical Package for the Social Sciences (SPSS) software (IBM SPSS Statistics 18, Inc., Chicago, IL). Descriptive data are reported as means and standard deviation (SD). Data normality was confirmed by the Shapiro-Wilk test. A two-way analysis of variance with repeated measures (time factor; pre-match and post-match) was used to compare the conditions (OM and $\mathrm{SM}$ ) and timepoints (pre and post). The Bonferroni test was used as a post hoc test. The level of significance was set at $p \leq 0.05$.

\section{Results}

$\mathrm{SIgA}$ responses to the $\mathrm{SM}$ and $\mathrm{OM}$ are presented in Figure 1. A significant decrease in SIgAabs (Figure 1A) was observed after OM when compared to pre-match value. In addition, a significant reduction in SIgArate (Figure 1B) was detected after $\mathrm{OM}$ when compared to pre-match value. There was no alteration for these variables when compared pre- and post-SM values. A significant increase in SIgArate was observed at pre-OM compared to pre-SM.

Figure 2 presents the magnitude of the ITL, which was assessed by a session RPE method and determined for SM and OM. A significantly higher ITL was observed for OM compared to SM.

A

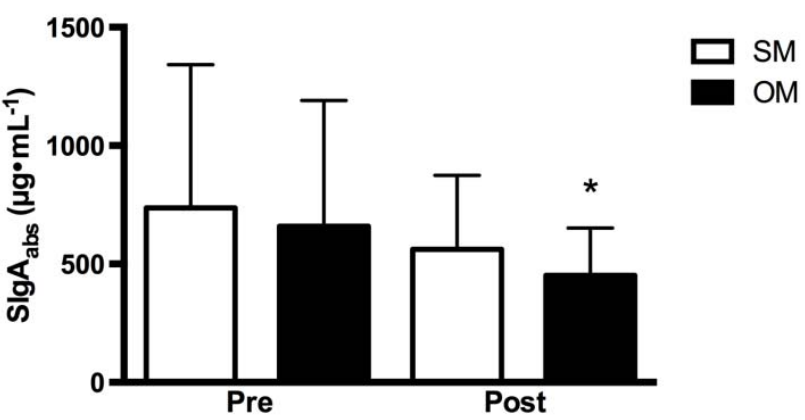

B

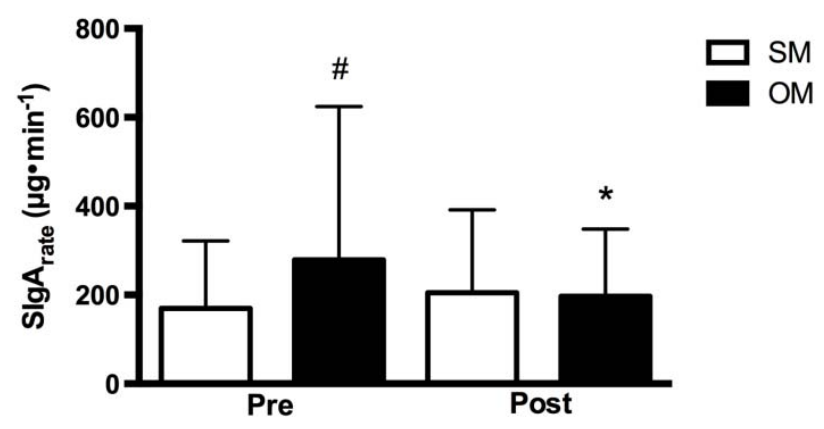

Figure 1

Salivary $\operatorname{Ig} A(\operatorname{SIg} A)$ responses (concentration $1 A$ and rate $1 B$ ) to simulated matches

$(S M)$ and official matches (OM). Note: \# - indicates significant difference from

$S M(p<0.05)$. * indicates significant difference from pre-match $(p<0.05)$. 


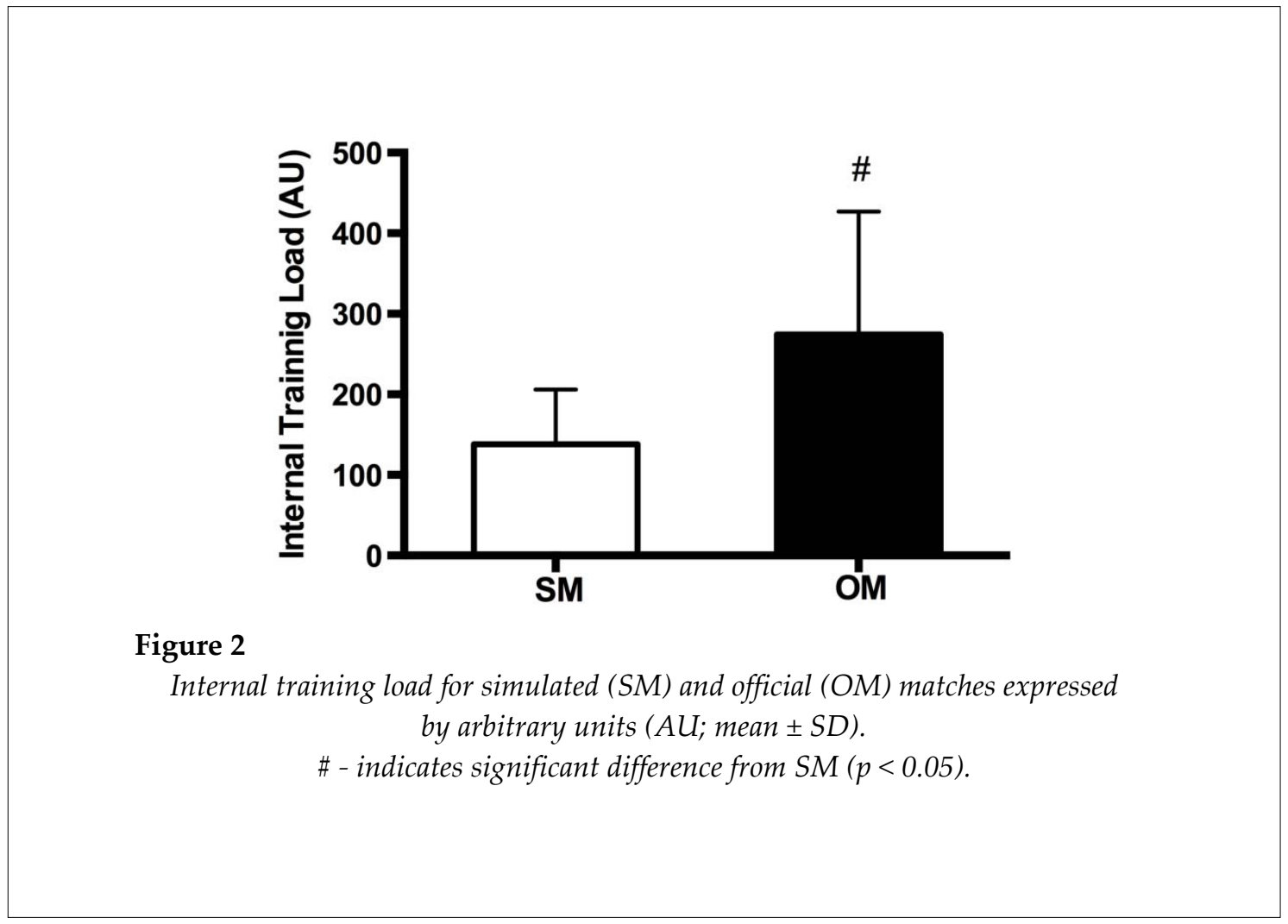

\section{Discussion}

The key findings of this study were: 1) a significant decrease in SIgA responses (SIgAabs and SIgArate) from pre to post-OM, and 2) a higher pre-OM SIgArate. The decrease in SIgA responses for $\mathrm{OM}$ is in line with the study's hypothesis which postulated that such a decrease from pre to post-OM would be observed. However, a higher pre-OM SIgArate was not expected. It is reasonable to speculate that the elevated psychological stress experienced before an official competition may influence the responses of mucosal immunity measures.

The absence of change in SIgA concentration from pre to post SM is in line with previously reported data on professional soccer players (Moreira et al., 2009), but differ from observations of Moreira et al. (2011b), Fredericks et al. (2012) and Owen et al. (2014). Moreira et al. (2009) demonstrated that both SIgAabs and SIgArate were not altered from pre to post soccer SM (two halves of $35 \mathrm{~min}$ with a $10 \mathrm{~min}$ interval). The authors suggested that some factors, such as withdrawal from the real competitive environment and the characteristics of the sport (intermittent character) might affect the responses. Together, the present results and findings reported by Moreira et al. (2009) indicate that SM do not represent a significant source of stress to modulate the mucosal immunity function of soccer players. The novelty of the present study is that its findings suggest that the same acute SIgA responses from SM may be expected regardless of the soccer player's age (adults or young soccer players). This result adds to the literature by advancing understanding of the effect of SM on mucosal immunity trends.

Contrary to the aforementioned findings, however, Fredericks et al. (2012) investigated the SIgA responses in English Premier League professional soccer players during the first training session of the season, which was completed after the summer off-season. They observed a decrease of SIgA/protein measure after $20 \mathrm{~min}$ of the end of the activity (Fredericks et al., 2012). One possible explanation for the differences between findings from that study and the results of the present study could be the fact that in the Fredericks et al.'s study the investigated players were returning from an off-training period, and the players in the present study and in the study 
of Moreira et al. (2009) were assessed during the competitive period. These discrepancies, therefore, could suggest that stress induced by $\mathrm{SM}$, training sessions or friendly matches, could be higher when players are not in their optimal fitness preparation state and could negatively affect the mucosal immune function.

Besides the fitness level, the characteristics and specific demands of the different team sports also appear to influence the acute SIgA responses. For instance, Moreira et al. (2011a) described a reduction in SIgA variables after SM in adult futsal players. The difference between the findings of this study and the results of the present investigation could be attributed to the distinct characteristics of the assessed team sports (soccer vs. futsal). Collectively, the findings of the aforementioned studies and the results of the present investigation suggest that the fitness level as well as the characteristics and demands of team sports should be taken into account for comparing results from different studies that have investigated acute SIgA responses.

Differentially from the responses to SM, in the present study, OM represented a sufficient source of stress that resulted in a significant decrement in SIgA concentration. This result suggests that the additional stress provided by participating in an official competition may lead to changes in the mucosal immunity function in young soccer players. These findings are in agreement with data from the study conducted on young female handball players (Nazem et al., 2011) that reported a decrement in SIgA concentration after matches perceived as "more difficult". Considering the results from the present study and the findings reported by Nazem et al. (2011), it is plausible to suggest that additional factors such as match intensity, competitive anxiety, pressure to perform, associated with the participation in $\mathrm{OM}$ (more important or even more difficult matches), may impose a higher level of stress on young team sport players, leading, therefore, to alteration in their mucosal immunity and then increasing the risk of URTI.

An inherent drawback of the present study could be the fact that the sampling period undertaken was not long enough to assess the relationship between the SIgA acute responses from OM and SM and the URTI incidence. While it could be viewed as a limitation of the study, it is noteworthy that the main aim of the present investigation was not to examine such a relationship, but to examine if mucosal immunity of youth players would be differently affected due to their participation in OM and SM. One could speculate that $\mathrm{OM}$ would impose a greater psychophysiological stress on players, leading to a decrease in SIgA. Since SIgA concentration and its change are used to predict susceptibility to infection, notably to URTI (Gleeson et al., 2012; Neville et al., 2008), and considering the fact that previous studies with young team sport athletes have demonstrated this relationship (He et al., 2010; Moreira et al., 2011b; Mortatti et al., 2012), it might be assumed from the findings of the present study that OM may negatively affect mucosal immunity of young soccer players leading to an elevated risk of URTI. On the other hand, it is important to highlight that while SIgA may be used as a useful and valid indicator of the mucosal immune function related to training loads or competitive demands, the relationship between this salivary marker and URTI symptoms might be not direct, mainly due to factors other than salivary antibody, which may contribute to infection development (Diamond et al., 2008).

It is noteworthy that a greater sessionRPE score was observed for OM when compared to SM. The session-RPE is a reliable method to assess exercise intensity in team sports (Impellizzeri et al., 2004; Manzi et al., 2010; Moreira et al., 2012c). Therefore, it is reasonable to assume that $\mathrm{OM}$ are played at higher intensity, probably as a result of a higher level of athletes' commitment. The effect of the intensity in SIgA responses was recently investigated by Owen et al. (2014). They compared 4 training sessions (low intensity, LI vs high intensity, HI) that were undertaken by 10 professional soccer players and showed that the percentage change from SIgA baseline concentration to post-training differed significantly between $\mathrm{HI}$ and LI, with a significant decrease in SIgA in the HI session (Owen et al., 2014). Indeed, previous studies reported a greater RPE score for real competition when compared to training simulations (Moreira et al., 2012a, 2012b, 2012c). The results from the present study suggest that the session-RPE method together with SIgA responses could be an insightful approach for coaches and coaching staff in order to assess the 
magnitude of training loads and competition demands which in turn could aid these professionals to adjust the training plan for young soccer player.

Another important finding of the present investigation was a higher SIgArate observed in pre-OM compared to pre-SM. Despite the difficulty to explain this result, it could be attributed to the increased sympathetic activity associated with the anticipatory stress of the official competition (Alix-Sy et al., 2008). This increased sympathetic activity would stimulate the SIgA production or even the translocation of this immunoglobulin through the epithelial cells (Bishop and Gleeson, 2009). Therefore, the higher SIgArate value would be associated with the enhanced psychological stress experienced by young players in anticipation of OM condition which in turn could amplify the sympathetic activity, that ultimately might lead to the increase in the production or in the translocation of SIgA elevating the available SIgA in the mucosal surface (Bishop and Gleeson, 2009).

During the pre-OM, there was no physical effort, therefore, the psychological stress may better justify the higher SIgArate value observed for this time-point. This phenomenon has been observed in many previous studies designed to observe the SIgA responses to different psychological sources of stress (Benham et al., 2009; Bosch et al., 2001; Willemsen et al., 2002; Zeier et al., 1996). In general, these studies have been developed to investigate the effect of psychological stress on SIgA without performance of physical effort. Previous findings indicate that an increase in SIgA concentration may be expected as a response to psychological challenges in the absence of physical effort (Benham et al., 2009; Bosch et al., 2001; Willemsen et al., 2002; Zeier et al., 1996). Collectively, these results strongly suggest that psychological stress may elevate SIgA concentration, and may explain, at least partially, the higher SIgArate value observed for pre-OM in the present study.

In summary, the results of the present study confirm the hypothesis that a decrease in SIgAabs and SIgArate after OM would be observed. Additionally, a greater internal training load was found for OM. Together, these results indicate that a higher psychophysiological stress would be expected for OM when compared to SM in young soccer players, which in turn may compromise the mucosal immunity of these athletes. Interestingly, a higher SIgArate at pre-OM compared to pre-SM was also noted. This is a novel result from the present study and it suggests that an increase in SIgA may be expected as a response to psychological challenges in the absence of physical effort. Coaches should be aware of the elevated psychophysiological stress induced by $\mathrm{OM}$ in young soccer players, as well as the postmatch compromised mucosal immunity, which might increase the risk of URTI. Therefore, coaches should consider these findings when planning training loads and recovery procedures to avoid or minimize the likelihood of URTI occurrences.

\section{Acknowledgements}

We would like to thank the FAPESP (Fundação de Amparo à Pesquisa do Estado de São Paulo; São Paulo Research Foundation, process, 2010/12489-6) for funding this research and for the scholarship offered to the first author, process, 2010/13084-0. We also wish to acknowledge the financial support of the CAPES Foundation, Ministry of Education of Brazil and thank all soccer players, their parents as well as team staff involved in this study for their committed participation.

\section{References}

Alix-Sy D, Le Scanff C, Filaire E. Psychophysiological responses in the pre-competition period in elite soccer players. J Sport Sci Med, 2008; 7: 446-454

Benham G, Nash MR, Baldwin DR. A comparison of changes in secretory immunoglobulin A following a stress-inducing and stress-reducing task. Stress Health, 2009; 25: 81-90

Bishop N, Gleeson M. Acute and chronic effects of exercise on markers of mucosal immunity. Front Biosci, 2009; 14: 4444-4456

Bosch JA, de Geus EJC, Kelder A, Veerman ECI, Hoogstraten J, Nieuw Amerongen AV. Differential effects of active versus passive coping on secretory immunity. Psychophysiology, 2001; 38: 836-846 
Diamond G, Beckloff N, Ryan LK. Host defense peptides in the oral cavity and the lung: similarities and differences. J Dent Res, 2008; 87: 915-927

Fahlman MM, Engels HJ. Mucosal IgA and URTI in American College Football Players: A Year Longitudinal Study. Med Sci Sport Exerc, 2005; 37: 374-380

Filaire E, Bonis J, Lac G. Relationship between psycological and physiological stress and salivary immunoglobulin A among female gymnasts. Percept Motor Skill, 2004; 99: 605-617

Foster C. Monitoring training in athletes with reference to overtraining syndrome. Med Sci Sport Exerc, 1998; 30: 1164-1168

Fredericks S, Fitzgerald L, Shaw G, Holt DW. Changes in salivary immunoglobulin A (IgA) following matchplay and training among English premiership footballers. Med J Malaysia, 2012; 67: 155-158

Gleeson M, Bishop N, Oliveira M, McCauley T, Tauler P, Muhamad AS. Respiratory infection risk in athletes: association with antigen-stimulated IL-10 production and salivary IgA secretion. Scan J Med Sci Spor, 2012; 22: 410-417

Gleeson M, Pyne DB. Exercise effects on mucosal immunity. Immunol Cell Biol, 2000; 78: 536-544

Hartwig TB, Naughton G, Searl J. Load, stress, and recovery in adolescent rugby union players during a competitive season. J Sport Sci, 2009; 27: 1087-1094

He CS, Tsai ML, Ko MH, Chang CK, Fang SH. Relationships among salivary immunoglobulin A, lactoferrin and cortisol in basketball players during a basketball season. Eur J Appl Physiol, 2010; 110: 989-995

Impellizzeri FM, Rampinini E, Coutts AJ, Sassi A, Marcora SM. Use of RPE-Based Training Load in Soccer. Med Sci Sport Exerc, 2004; 36: 1042-1047

Koch AJ, Wherry AD, Petersen MC, Johnson JC, Stuart MK, Sexton WL. Salivary immunoglobulin A response to a collegiate rugby game. J Strength Cond Res, 2007; 21: 86-90

Li TL, Lin HC, Ko MH, Chang CK, Fang SH. Effects of prolonged intensive training on the resting levels of salivary immunoglobulin A and cortisol in adolescent volleyball players. J Sports Med Phys Fitness, 2012; 52: 569-573

Mackinnon LT. Immunoglobulin, antibody and exercise. Exerc Immunol Rev, 1996; 2: 1-35

Manzi V, D'Ottavio S, Impellizzeri FM, Chaouachi A, Chamari K, Castagna C. Profile of weekly training load in elite male professional basketball players. J Strength Cond Res, 2010; 24: 1399-1406

Moreira A, Arsati F, Cury PR, Franciscon C, Oliveira PR, de Araújo VC. Salivary immunoglobulin a response to a match in top-level brazilian soccer players. J Strength Cond Res, 2009; 23: 1968-1973

Moreira A, Arsati F, de Oliveira Lima-Arsati YB, Freitas CG, de Araújo VC. Salivary immunoglobulin A responses in professional top-level futsal players. J Strength Cond Res, 2011a; 25: 1932-1936

Moreira A, Arsati F, De Oliveira Lima-Arsati YB, Simões AC, De Araújo VC. Monitoring stress tolerance and occurrences of upper respiratory illness in basketball players by means of psychometric tools and salivary biomarkers. Stress and health, 2011b; 27: 166-172.

Moreira A, Bacurau RF, Napimoga MH, Arruda AFS, Freitas CG, Drago G, Aoki MS. Salivary IL-21 and IgA responses to a competitive match in elite basketball players. Biol Sport, 2013a; 30: 243-247

Moreira A, Crewther B, Freitas CG, Arruda AFS, Costa EC, Aoki MS. Session RPE and salivary immuneendocrine responses to simulated and official basketball matches in elite young male athletes. J Sports Med Phys Fit, 2012a; 52: 682-687

Moreira A, Franchini E, Freitas CG, Schultz de Arruda AF, Moura NR, Costa EC, Aoki MS. Salivary cortisol and immunoglobulin A responses to simulated and official Jiu-Jitsu matches. J Strength Cond Res, 2012b; 26: 2185-2191

Moreira A, Freitas CG, Nakamura FY, Drago G, Drago M, Aoki MS. Effect of match importance on salivary cortisol and immunoglobulin A responses in elite young volleyball players. J Strength Cond Res, 2013b; 27: 202-207

Moreira A, McGuigan M, Arruda AFS, Freitas CG, Aoki MS. Monitoring internal load parameters during simulated and official basketball matches. J Strength Cond Res, 2012c; 26: 861-866

Moreira A, Mortatti AL, Arruda AFS, Freitas CG, de Arruda M, Aoki MS. Salivary IgA response and upper respiratory tract infection symptoms during a 21-week competitive season in young soccer players. $J$ Strength Cond Res, 2014; 28: 467-473 
Mortatti AL, Moreira A, Aoki MS, Crewther BT, Castagna C, Arruda AFS, Filho JM. Effect of Competition on Salivary Cortisol, Immunoglobulin A, and Upper Respiratory Tract Infections in Elite Young Soccer Players. J Strength Cond Res, 2012; 26: 1396-1401

Nazem G, Sharifi GR, Taghian F, Jourkesh M, Ostojic SM, Calleja-Gonzalez J, Keikhai BM. The effects of successive official competitions on salivary cortisol and immunoglobulin responses in women handballers. Serbian J Sport Sci, 2011; 5: 67-73

Neville V, Gleeson M, Folland JP. Salivary IgA as a risk factor for upper respiratory infections in elite professional athletes. Med Sci Sport Exerc, 2008; 40: 1228-1236

Nieman DC, Kernodle MW, Henson DA, Sonnenfeld G, Morton DS. The acute response of the immune system to tennis drills in adolescent athletes. Res Q Exercise Sport, 2000; 71: 403-408

Owen AL1, Wong DP, Dunlop G, Groussard C, Kebsi W, Dellal A, Morgans R, Zouhal H. High intensity training and salivary immunoglobulin-A responses in professional top-level soccer players: effect of training intensity. J Strength Cond Res, 2014; Jan 19 (epub ahead of print)

Willemsen G, Carroll D, Ring C, Drayson M. Cellular and mucosal immune reactions to mental and cold stress: Associations with gender and cardiovascular reactivity. Psychophysiology, 2002; 39: 222-228

Zeier H, Brauchli P, Joller-Jemelka HI. Effects of work demands on immunoglobulin and cortisol in air traffic controllers. Biol Psychol, 1996; 42: 413-423

\section{Corresponding author:}

\section{Alexandre Moreira ${ }^{a}$}

Av. Prof. Mello de Moraes, 65 - Cidade Universitária. CEP: 05508-030. São Paulo, SP - Brazil;

Phone: 551130918789

E-mail address: alemoreira@usp.br 